\title{
Special issue on micro-UAV perception and control
}

\author{
Nathan Michael · Davide Scaramuzza • Vijay Kumar
}

Received: 17 April 2012 / Accepted: 26 April 2012 / Published online: 5 May 2012

(C) Springer Science+Business Media, LLC 2012

This special journal issue focuses on the problems of perception and control for micro unmanned aerial vehicles (UAVs). These two topic areas are particularly important when considering autonomous micro-UAVs capable of operating in complex and challenging environments. A fundamental limitation of micro-UAVs arises from the onboard power for actuation, sensing, and computation, which can be insubstantial because of limitations on specific power and energy density with current of-the-shelf energy sources. The choice of mechanical design determines the permissible payload, which in turn dictates the onboard sensing and computation. Hence, micro-UAV perception and control are tightly coupled.

The solution to such perception problems as 3D state estimation and mapping can be computationally demanding due to the need for the continual processing of rich 3D sensor observations. Further, low-mass and small-scale sensors and processors capable of providing and operating on the required 3D information are not readily available. Therefore, researchers must consider sensor and processor availability and design along with optimized algorithms to enable perception on micro-UAVs.

The control of micro-UAVs requires careful modeling of the vehicle's dynamics and operating constraints such as actuation and sensor saturation. The small size also magnifies

N. Michael $(\bowtie) \cdot$ V. Kumar

University of Pennsylvania, Philadelphia, PA, USA

e-mail: nmichael@grasp.upenn.edu

V. Kumar

e-mail:kumar@grasp.upenn.edu

D. Scaramuzza

University of Zurich, Zurich, Switzerland

e-mail: davide.scaramuzza@ieee.org the effects of external interactions and model uncertainty. Therefore, it is necessary to pursue trajectory and feedback control design strategies that are robust and adaptive to these concerns.

This issue focuses primarily on rotorcraft micro-UAVs (helicopters and quadrotors) with papers on sensor and vehicle design, state estimation and 3D mapping, trajectory and feedback control design, learning and nonlinear model adaptation, and control stability given external interactions. A brief overview of each of the papers follows.

The paper by Roberts et al. (doi:10.1007/s10514-0129277-0) details the design of a novel infrared-based 3D relative positioning and proximity sensor to enable spatial coordination between multiple aerial vehicles. A precisely arranged ring of emitters and photodiodes is used to detect the relative range, bearing, and elevation between sensors. A turn-taking algorithm is employed to avoid signal interference between multiple transmissions-ensuring effective operation when multiple robots fly in close proximity. The physical design and integration on a flying is reviewed. Experimental performance and robustness evaluations detail the accuracy and operating characteristics of the sensor in variable ambient light conditions.

The paper by Meier et al. (doi:10.1007/s10514-0129281-4) reviews the hardware and software design of an open-source quadrotor equipped with customized onboard computation and algorithms enabling vision-based localization and navigation. The vehicle is equipped with multiple stereo cameras to permit real-time vision-based localization and obstacle collision avoidance. The vehicle localizes with respect to the environment by detecting markers and computing local changes in position and orientation with respect to these markers. This relative transformation estimate is fused with inertial measurement unit (IMU) readings via a discrete Kalman Filter prior to use in a proportional-integral- 
derivative (PID) controller. Obstacles are detected based on $3 \mathrm{D}$ point cloud generated from the depth information provided by forward facing stereo cameras. The software architecture and implementation, hardware characteristics, and vehicle capabilities are detailed and compared to existing commercially available platforms. System performance and localization accuracy are analyzed through experimental studies.

The paper by Ghadiok et al. (doi:10.1007/s10514-0129286-z) presents the hardware, software, and algorithms required to develop an autonomous quadrotor capable of vision-based navigation and aerial gripping. The system design discussion details low-cost off-the-shelf components and includes an assembly and architecture overview. Onboard feedback control and state estimation is accomplished via a modified PID controller and a nonlinear complimentary filter that fuses IMU information with relative transformation estimates resulting from vision-based simultaneous localization and mapping, respectively. Experimental results analyze the accuracy and robustness of both the onboard control and estimation in indoor and outdoor environments and when the vehicle is experiencing disturbances due to external effects. Two representative applications of the vehicle are demonstrated in experimentation: perching on an inclined surface using vision to detect the perching surface and aerial gripping (including details on the mechanical design of the gripper).

The paper by Hehn et al. (doi:10.1007/s10514-0129282-3) considers the fundamental performance capabilities of a quadrotor by determining time-optimal trajectories given the saturation of the vehicle's actuation and onboard sensing. Pontryagin's minimum principle is used to develop time-optimal maneuvers via bang-bang control inputs which can be composed to generate optimal trajectories. The existence of such optimal trajectories is shown to always exist between two arbitrary states and an algorithm is proposed to compute these trajectories based on the composition of timeoptimal maneuvers. Illustrations of maneuvers in simulation along with performance comparisons with linear controllers demonstrate the optimally of the approach. Experimental results provide insight into the correspondance between the expected and actual performance despite inaccuracies in vehicle modeling.

The paper by Lupashin and D'Andrea (doi:10.1007/ s10514-012-9289-9) proposes a method for designing aggressive trajectories for quadrotors through the composition of maneuvers and online adaptation to account for model parameter inaccuracies and actuation and sensing limitations. A general initial trajectory is proposed based on a desired predefined trajectory structure composed of time and model parameterized maneuvers. An iterative adaptation procedure is applied that optimizes over the system and trajectory parameters that minimizes the error between the desired and actual final state of the vehicle given an analytic model of the system. Maneuver design based on time-optimal control techniques and methods for implementation provide insight into the application of the approach. Experimental studies leveraging the adaptation procedure and a baseline comparisons to theoretically predicted time-optimal trajectories illustrate the effectiveness of these techniques for several representative applications.

The paper by Schoellig et al. (doi:10.1007/s10514-0129283-2) considers the problem of estimating model parameters and disturbances while tracking a desired trajectory via iterative learning-based methods with application to a quadrotor. The iterative learning control technique is cast as an optimization problem linearized about the desired trajectory based on the vehicle dynamic model and a lifteddomain representation. A Kalman filter based disturbance estimator is incorporated into the learning update along with a technique for extending the optimization horizon. Considerations specific to the dynamic model of a quadrotor with actuation and sensing constraints and the design of feasible trajectories enable the application of the approach in experimentation. Three experimental studies investigate the effectiveness of the approach to accurately identify uncertain model parameters and enable a quadrotor to track a desired trajectory.

The paper by Pounds et al. (doi:10.1007/s10514-0129280-5) analyzes the stability of helicopter and quadrotor UAVs carrying external payloads. The regions of stability and dynamical response when applying sustained and instantaneous off-center loads is considered for both systems. The impact and consequences of loads on the controllability of a helicopter given off-the-shelf PID flight controllers is investigated via sensitivity analysis in numerical simulations. The models and analysis are validated experimentally on a small-scale helicopter UAV.

The paper by Turpin et al. (doi:10.1007/s10514-0129279-y) focuses on designing trajectories and decentralized feedback control laws that drive a team of quadrotors to converge to a desired formation shape while maintaining robustness to inter-robot communication failures. The individual vehicle model and trajectory generation and tracking control laws are detailed before discussing the modeling of the robot formation. A finite-horizon decentralized leaderfollower formation control law is proposed that guarantees convergence to a desired formation shape through consensus on trajectories of neighboring robots. The formation control law is shown to be convergent to the desired shape. Numerical simulations and experiments investigate the performance of the approach for teams of quadrotors moving in formation. The robustness of the formation control law to communication failures is studied in experimentation by considering the formation error in convergence to the desired shape while undergoing simulated dropped packets between robots. 
The paper by Eynard et al. (doi:10.1007/s10514-0129285-0) presents a real-time stereo-vision based approach to localization for a micro-UAV equipped with downward facing fisheye and perspective cameras. The approach first determines an attitude estimate by horizon and vanishing point detection. The altitude estimate is computed by planesweeping and the known correspondance of the detected ground plane between the spherical and perspective models. Translational motion is determined based on the estimated altitude and observed changes in features in the environment and on the ground. Performance comparisons of the algorithm accuracy and efficiency when executed on an external system and via an onboard embedded mobile processor provide insight into the applicability of the approach on micro-UAVs.

The paper by Doitsidis et al. (doi:10.1007/s10514-0129292-1) details an approach to multi-UAV optimal surveillance and coverage based on terrain elevation maps generated from observations made by cameras on each quadrotor UAV. The approach leverages previous localization and mapping results via vision-based methods with discussion of pertinent hardware, software, and implementation details. Given the state of each robot, a 3D point cloud is generated from image features and associated triangulated positions. A Delaunay triangulation of this point cloud is performed online to create a terrain elevation map of the environment. The optimal surveillance and coverage control pol- icy of each robot is computed using an adaptive optimization algorithm that seeks to maximize the terrain coverage while ensuring cooperation between vehicles. Experimental evaluation in two environments with teams of UAVs provides insight into the algorithmic approach and presents the resulting vehicle deployments and terrain-elevation maps.

The paper by Scherer et al. (doi:10.1007/s10514-0129293-0) focuses on micro-UAV state estimation and mapping in river environments. The vehicle state is first estimated from IMU, GPS (when available), and stereo visual odometry observations. This estimate coupled with observations from a panning laser enables the generation of rich 3D maps. A sparse pose-graph formulation leveraging a sliding window technique reduces the required computation for online processing. Obstacle maps for vehicle planning and navigation are generated via a distance transform algorithm that requires limited CPU overhead. An automated visionbased detection algorithm based on self-supervised training enables the detection, labeling, and mapping of the river surface. Experimental studies in a river environment report on the effectiveness of the methods and repeatability as the environment changes.

These papers highlight both strong theoretical and experimental studies and exemplify the rich research currently ongoing in this area. We hope you enjoy the papers! 